\title{
CONTROLE EXTREMAL ESTOCÁSTICO NA PRESENÇA DE ATRASOS
}

\author{
Paulo Cesar Souza da Silva* Tiago Roux Oliveira* \\ * Programa de Pós-Graduação em Engenharia Eletrônica, \\ Universidade do Estado do Rio de Janeiro (UERJ), Rio de Janeiro - \\ RJ,(e-mail: cesar.paulo151@hotmail.com.br, tiagoroux@uerj.br)
}

\begin{abstract}
This work presents a control design and analysis for a scalar Newton-based stochastic extremum seeking control under input-output delays. A new predictor with stochastic sinusoidal pertubation-based estimate of the Hessian's inverse is incorporated in the closed-loop such that the convergence rate of the controller in real-time can be made developer-assignable. Exponential stability and convergence to a small neighborhood of the unknown extremum point can be obtained. This result is rigorously reached by using backstepping transformation and averaging in infinite dimensions. A numerical example is shown to present the effectiveness of the proposed predictor-based stochastic extremum seeking for time-delay compensation.

Resumo: Neste artigo, é proposto um projeto de controle por busca extremal estocástica baseado no algoritmo de Newton na presença de atrasos de entrada e saída. Um novo preditor com uma estimativa da inversa da Hessiana baseada em perturbações senoidais estocásticas (sinais de dither) é incorporado à malha fechada, de modo que a taxa de convergência do controlador em tempo real possa ser especificada pelo usuário. A estabilidade exponencial e convergência a uma pequena vizinhança do ponto de extremo é obtida. Este resultado é rigorosamente alcançado utilizando a transformação backstepping e teoria da média em dimensões infinitas. Um exemplo numérico é apresentado para expressar a eficiência do controlador extremal estocástico baseado em preditor para compensação de atrasos.
\end{abstract}

Keywords: Delays; Predictors; Stochastic Extremum Seeking; Newton Method; Backstepping Transformation.

Palavras-chaves: Atrasos; Preditores; Controle Extremal Estocástico; Método de Newton; Transformação Backstepping.

\section{INTRODUÇÃO}

Apesar do grande número de publicações sobre controle por busca extremal (Extremum Seeking Control - ESC) (Krstic (2014); Adetola and Guay (2007); Ghaffari et al. (2012); Oliveira et al. (2011)), não há um artigo que encare o desafio do ESC estocástico (Liu and Krstic (2012)) baseado nos algoritmos do tipo Gradiente e de Newton com a inserção de atrasos na entrada e saída (Oliveira et al. (2017)), respectivamente. Vale ressaltar que as principais vantagens do controlador extremal estocástico sobre o controlador extremal determinístico (com sinais de excitação determinísticos) são a possibilidade de escapar dos extremos locais, bem como a garantia de uma taxa de convergência mais rápida (Liu and Krstic (2012)).

$\mathrm{Na}$ ausência de atrasos, o método de Newton possui algumas vantagens (Krstic (2014); Ghaffari et al. (2012)), que são: apresentar taxas de convergência mais rápidas e remover a dependência da taxa de convergência da segunda derivada desconhecida (Hessiana) do mapeamento nãolinear a ser otimizado, sendo arbitrariamente atribuída ao

\footnotetext{
^ P.C.S. Silva e T.R. Oliveira agradecem CNPq, CAPES e FAPERJ pelo suporte financeiro.
}

desenvolvedor/usuário. A garantia dessa propriedade é o objetivo desse trabalho.

Além disso, cabe comentar que a remoção da dependência da taxa de convergência da Hessiana é um problema bem desafiador, já que o ESC está relacionado com uma boa taxa de convergência, enquanto que os atrasos quando são inseridos em um sistema de malha fechada e simplesmente ignorados, restringem severamente a taxa de convergência do sistema como um todo ou leva o sistema à instabilidade (da literatura sabe-se que o controle extremal não é robusto à presença de atrasos). Em (Oliveira et al. (2017)), foi o início da exploração do ESC determinístico com a inserção dos atrasos de entrada e/ou saída. Desse modo, existe o desafio de realizar-se um estudo eficiente e inovador que pesquise os algoritmos do controle extremal no meio estocástico, mais uma vez inserindo os atrasos nos sinais de entrada e saída do sistema e fazendo o uso do preditor realimentado.

Dessa maneira, a solução encontrada neste artigo para o problema exposto é dada empregando-se uma realimentação por preditor com uma estimativa da inversa da Hessiana desconhecida baseada na perturbação senoidal estocástica (Liu and Krstic (2012)). A análise de estabilidade é rigorosamente construída via transformação backstepping 


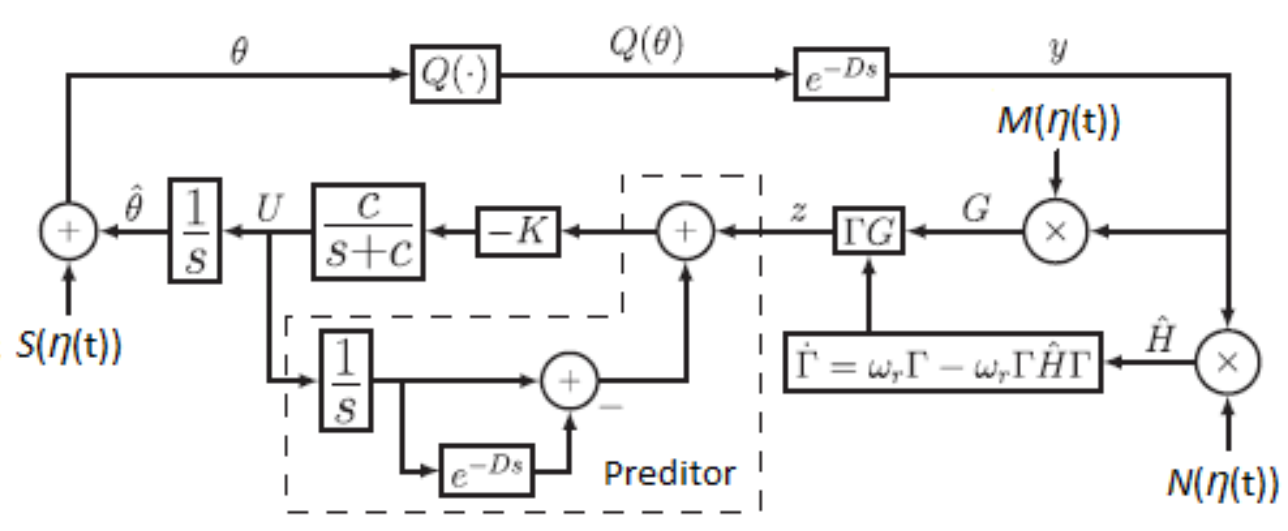

Figura 1. Diagrama de blocos do esquema de predição para compensação dos atrasos na saída por busca extremal estocástica empregando o algoritmo de Newton. A realimentação por preditor com uma estimativa inversa da Hessiana baseada em perturbações estocásticas obedece as equações (14), (15) e (26) e os sinais de dither são dados por: $S(\eta(t))=\operatorname{asen}(\eta(t+D)), M(\eta(t))=\frac{2}{a} \operatorname{sen}(\eta(t))$ e sinal de demodulação $N(\eta(t))=-\frac{8}{a^{2}} \cos (2 \eta(t))$.

(Krstic (2009)) e a teoria da média em dimensões infinitas (Hale and Lunel (1990); Lehman (2002)), capturando-se o estado de dimensão infinita devido aos atrasos.

Na seção 2, há a introdução do projeto do preditor para a compensação dos atrasos no ESC estocástico baseado no algoritmo de Newton. Na seção 3, demonstra-se a estabilidade exponencial e a convergência em tempo real à uma pequena vizinhança do extremo desejado. Na seção 4, os resultados da simulação são apresentados com a finalidade de demonstrar a aplicabilidade da proposta de compensação do atraso na busca extremal estocástica. $\mathrm{Na}$ seção 5, o artigo é finalizado.

Notação: A derivada parcial de $u(x, t)$ é denotada por $u_{t}(x, t)$ e $u_{x}(x, t)$ ou, ocasionalmente, por $\partial_{t} u_{a v}(x, t) \mathrm{e}$ $\partial_{x} u_{a v}(x, t)$ para se referir ao operador do sinal médio $u_{a v}(x, t)$. Admitindo-se um sistema não-linear genérico $\dot{x}=f(t, x, \epsilon)$, onde $x \in R^{n}, f(t, x, \epsilon)$ é periódico em t com período $T$, isto é, $f(t+T, x, \epsilon)=f(t, x, \epsilon)$. Então, para $\epsilon>0$ suficientemente pequeno, é possível obter o modelo médio dado por $\dot{x}=f_{a v}\left(x_{a v}\right)$, com $f_{a v}=$ $\frac{1}{T} \int_{0}^{T} f\left(\tau, x_{a v}, 0\right) d \tau$, onde $x_{a v}(t)$ denota a versão média do estado $x(t)$ (Khalil, 2002). Conforme definido em (Khalil, 2002), uma função vetorial $f(t, \epsilon) \in R^{n}$ é dita de ordem $O(\epsilon)$ dentro do intervalo $[t 1, t 2]$ se existem constantes positivas $k$ e $\epsilon^{*}$ tais que $|f(t, \epsilon)| \leq k \epsilon \forall \epsilon \in\left[0, \epsilon^{*}\right]$ e $\forall t \in$ $[t 1, t 2]$. Por vezes, estimativas para as constantes $k$ e $\epsilon^{*}$ serão fornecidas, podendo-se quantificar a correspondente aproximação $O(\epsilon)$. Caso contrário, $O(\epsilon)$ será satisfeito sendo uma ordem da relação de magnitude válida para $\epsilon$ suficientemente pequeno.

\section{ESC ESTOCÁSTICO BASEADO NO MÉTODO DE NEWTON PARA SISTEMAS COM ATRASOS}

O ESC escalar apresenta aplicação na qual o objetivo é maximizar ou minimizar a saída $y \in R$ de um mapeamento estático não-linear $Q(\theta)$ desconhecido através da variação em tempo-real da entrada $\theta \in R$. Aqui, considera-se adicionalmente que existe um atraso constante e desconhecido $D$ no caminho de atuação ou no sistema de medição, tal que a saída medida é dada por:

$$
y(t)=Q(\theta(t-D)) .
$$

Para o melhor entendimento da notação, assume-se ao longo do artigo que o sistema tem apenas a saída atrasada conforme visto na Figura 1. Os resultados encontrados neste artigo podem ser diretamente estendidos ao caso de entrada atrasada, uma vez que qualquer atraso de entrada possa ser direcionado para a saída do mapeamento estático. O caso no qual os atrasos de entrada $D_{i n}$ e de saída $D_{\text {out }}$ ocorrem simultaneamente também pode ser tratado assumindo-se que o atraso total a ser neutralizado seja $D=D_{\text {in }}+D_{\text {out }}, \operatorname{com} D_{\text {in }}, D_{\text {out }} \geq 0$.

Sem perda de generalidade, assume-se o problema de busca pelo máximo, de tal forma que o valor de $\theta$ que maximiza $y$ é denotado por $\theta^{*}$. Por simplicidade, considera-se que o mapeamento escalar não-linear quadrático é da forma:

$$
Q(\theta)=y^{*}+\frac{H}{2}\left(\theta-\theta^{*}\right)^{2},
$$

no qual além das constantes $\theta^{*} \in R$ e $y^{*} \in R$ serem desconhecidas, o escalar $H<0$ é a Hessiana desconhecida do mapeamento estático.

Na Figura 1, exemplifica-se a versão escalar para o controle por busca extremal estocástica baseado no algoritmo de Newton empregando a realimentação por preditor para a compensação dos atrasos.

\subsection{Sinais e sistemas}

Substituindo-se (2) em (1), obtém-se o mapeamento quadrático e estático com atraso de interesse:

$$
y(t)=y^{*}+\frac{H}{2}\left(\theta(t-D)-\theta^{*}\right)^{2} .
$$

Seja $\hat{\theta}$ a estimativa de $\theta^{*} \mathrm{e}$

$$
\tilde{\theta}(t)=\hat{\theta}(t)-\theta^{*}
$$


o erro de estimativa. Da Figura 1, tem-se que $\dot{\hat{\theta}}(t)=U(t)$ e conclui-se que a dinâmica do erro pode ser escrita da seguinte maneira (atrasando-se em $D$ ambos os lados da equação resultante):

$$
\dot{\tilde{\theta}}(t-D)=U(t-D)
$$

Além disso, tem-se

$$
G(t)=M(\eta(t)) y(t), \theta(t)=\hat{\theta}(t)+S(\eta(t)),
$$

em que os sinais de perturbação senoidais (chamados de sinais de dither) são dados por:

$$
S(\eta(t))=\operatorname{asen}(\eta(t+D)), \quad M(\eta(t))=\frac{2}{a} \operatorname{sen}(\eta(t)),
$$

com amplitude $a$ e frequência $\omega$ não-nulas. Empregam-se perturbações senoidais estocásticas via processo de Wiener sobre o limite de um círculo (Liu and Krstic, 2012; Mills and Krstic, 2018),

$$
\theta(t)=\hat{\theta}(t)+a \operatorname{sen}(\eta(t+D))
$$

onde

$$
\eta(t)=\omega \pi\left(1+\operatorname{sen}\left(W_{\omega t}\right)\right)
$$

representa um processo de Markov homogeneamente ergódico e utilizando-se a regra da cadeia estocástica e , obtémse:

$$
d \eta=-\omega \frac{\pi}{2} \operatorname{sen}\left(W_{\omega t}\right) d t+\omega \pi \cos \left(W_{\omega t}\right) d W_{\omega t} .
$$

uma vez que o atraso na saída pode ser transferido para a saída do integrador com a finalidade de análise ou equivalentemente para a entrada do sistema, então, o deslocamento de fase $+D$ é aplicado para compensar o efeito do atraso no sinal de dither aditivo em (7). A estimativa da Hessiana $H$ desconhecida é

$$
\hat{H}(t)=N(\eta(t)) y(t)
$$

cujo sinal de demodulação é dado por:

$$
N(\eta(t))=-\frac{8}{a^{2}} \cos (2 \eta(t))
$$

Em Ghaffari et al. (2012); Liu and Krstic (2012), foi provado que

$$
\frac{1}{\Pi} \int_{0}^{\Pi} N(\sigma) y d \sigma=H, \quad \Pi=2 \pi / \omega,
$$

se um mapeamento quadrático com em (2) é considerado. Além disso, a versão média de $H(t)$ é dada por $\hat{H}_{a v}(t)=$ $(N y)_{a v}(t)=H$. Define-se o sinal mensurável:

$$
z(t)=\Gamma(t) G(t)
$$

em que $\Gamma(t)$ é atualizado pela seguinte equação diferencial de Riccati (Ghaffari et al., 2012):

$$
\dot{\Gamma}=\omega_{r} \Gamma-\omega_{r} \hat{H} \Gamma^{2},
$$

com $\omega_{r}>0$ sendo uma constante de projeto. A equação (15) gera uma estimativa da inversa da Hessiana $\left(H^{-1}\right)$, evitando-se assim inversões da Hessiana estimada, que durante a fase transitória pode ser zero. $\mathrm{O}$ erro de estimação da inversa da Hessiana pode ser definido como:

$$
\tilde{\Gamma}(t)=\Gamma(t)-H^{-1}
$$

e sua equação dinâmica pode ser alcançada a partir de (15) e (16) como

$$
\dot{\tilde{\Gamma}}=\omega_{r}\left[\tilde{\Gamma}+H^{-1}\right] \times\left[1-\hat{H}\left(\tilde{\Gamma}+H^{-1}\right)\right] .
$$

2.2 Realimentação via preditor através da estimativa inversa da Hessiana

Pela análise da média (Ghaffari et al., 2012; Liu and Krstic, 2012), pode-se verificar de $G(t)$ em (6) e $z(t)$ em (14) que

$$
z_{a v}(t)=\Gamma_{a v}(t) H \tilde{\theta}_{a v}(t-D) .
$$

De (16), a equação (18) pode ser escrita em termos de $\tilde{\Gamma}_{a v}(t)=\Gamma_{a v}(t)-H^{-1}$ como:

$$
z_{a v}(t)=\tilde{\theta}_{a v}(t-D)+\tilde{\Gamma}_{a v}(t) H \tilde{\theta}_{a v}(t-D) .
$$

O segundo termo, no lado direito de (19) é quadrático em $\left(\tilde{\Gamma}_{a v}, \tilde{\theta}_{a v}\right)$, então a linearização de $\Gamma_{a v}(\mathrm{t})$ em $H^{-1}$ resulta em uma versão linearizada dada por

$$
z_{a v}(t)=\tilde{\theta}_{a v}(t-D)
$$

De (5) e (20), os seguintes modelos médios podem ser conseguidos

$$
\begin{gathered}
\dot{\tilde{\theta}}_{a v}(t-D)=U_{a v}(t-D) \\
\dot{z}_{a v}(t)=U_{a v}(t-D) .
\end{gathered}
$$

onde $U_{a v} \in R$ é o controle médio resultante de $U \in R$.

Com o objetivo de motivar o projeto da realimentação por preditor, a ideia aqui consiste em compensar o atraso através do estado futuro $z(t+D)$, ou $z_{a v}(t+D)$ na versão média equivalente do sistema. Para se obter $z_{a v}(t+D)$ com a fórmula da variação das constantes para (21) e (22), o estado futuro é dado por

$$
z_{a v}(t+D)=z_{a v}(t)+\int_{t-D}^{t} U_{a v}(\sigma) d \sigma
$$

onde o sinal de controle $U_{a v}(\sigma)$ é calculado na janela de tempo passada $[t-D, t]$. Dado qualquer ganho estabilizante $k>0$, o controle médio deve ser dado por 


$$
U_{a v}(t)=-k\left[z_{a v}(t)+\int_{t-D}^{t} U_{a v}(\sigma) d \sigma\right],
$$

resultando no controle médio $U_{a v}(t)=-k z_{a v}(t+D), \forall t>$ 0 , como desejado. Desse modo, o sistema médio deve ser, $\forall t \geq D$ :

$$
\frac{d \tilde{\theta}_{a v}(t)}{d t}=-k \tilde{\theta}_{a v}(t)-k \tilde{\Gamma}_{a v}(t+D) H \tilde{\theta}_{a v}(t) .
$$

Uma vez que $k \tilde{\Gamma}_{a v} H \tilde{\theta}_{a v}$ é quadrático em $\left(\tilde{\Gamma}_{a v}, \tilde{\Gamma}_{a v}\right)$, a linearização do sistema (25) tem autovalor determinado por $-k$. Então, a estabilidade exponencial local do algoritmo do tipo de Newton pode ser garantida com a taxa de convergência, que é independente da Hessiana $H$ desconhecida.

Em (Oliveira and Krstic, 2015), mostra-se que o objetivo de controle pode ainda ser alcançado se uma modificação no controlador baseado em preditor, que emprega um filtro passa-baixas, é aplicada tal que o teorema da média (Averaging Theorem) em dimensões infinitas (Hale and Lunel, 1990; Lehman, 2002) possa ser invocado. Nesse sentido, é proposto a seguinte compensação por preditor filtrado Krstic (2008):

$$
U(t)=\frac{c}{s+c}\left\{-k\left[z(t)+\int_{t-D}^{t} U(\tau) d \tau\right]\right\},
$$

em que $c>0$ é suficientemente grande, i.e., a realimentação por preditor é da forma de uma filtragem passa-baixas da versão não-média de (24). Utiliza-se a notação mista do domínio do tempo e da frequência em (26), para demonstrar que a função de transferência age como um operador em uma função no domínio do tempo. A realimentação por preditor (26) é baseada em perturbação média porque $z$ em (14) é atualizado de acordo com a estimativa $\Gamma$ para a inversa da Hessiana desconhecida $H^{-1}$ dada por (15), com $\hat{H}(t)$ em (11) satisfazendo a propriedade da média (13).

\section{ANÁLISE DE ESTABILIDADE}

O principal resultado de estabilidade/convergência para o sistema em malha fechada é dado a seguir. Os operadores $\mathbf{E}\{\cdot\}$ e $\mathbf{P}\{\cdot\}$ denotam respectivamente, o valor esperado e a probabilidade dos sinais.

Teorema 1 Considere o sistema em malha fechada da Figura 1, com saída atrasada (3). Existe $c^{*}>0$ tal que, $\forall c \geq c^{*}, \exists \omega^{*}(c)$ de maneira tal que $\forall \omega \geq \omega^{*}$, o sistema atrasado em malha fechada (5) e (26), com $z(t)$ em (14), $G(t)$ em $(6), \Gamma(t)$ em (15) e estado $\tilde{\Gamma}(t), \tilde{\theta}(t-D), U(\sigma)$, $\forall \sigma \in[t-D, t]$, tem uma solução periódica localmente exponencialmente estável em $t$ de período $\Pi=2 \pi / \omega$, denotada por $\tilde{\Gamma}^{\Pi}(t), \tilde{\theta}^{\Pi}(t-D), U^{\Pi}(\sigma), \forall \sigma \in[t-D, t]$, satisfazendo $\forall t \geq 0$ :

$$
\begin{aligned}
\mathbf{E}\left\{\left|\tilde{\Gamma}^{\Pi}(t)\right|^{2}+\left|\tilde{\theta}^{\Pi}(t-D)\right|^{2}+\left[U^{\Pi}(t)\right]^{2}+\right. & \\
& {\left.\left[\int_{t-D}^{t} U^{\Pi}(\sigma)\right]^{2} d \sigma\right\}^{1 / 2}=O(1 / \omega) }
\end{aligned}
$$

Além do mais,

$$
\begin{aligned}
& \lim _{(1 / \omega) \rightarrow 0} \mathbf{P}\left\{\lim _{t \rightarrow \infty} \sup \left|\theta(t)-\theta^{*}\right|\right\}=O(a+1 / \omega), \\
& \lim _{(1 / \omega) \rightarrow 0} \mathbf{P}\left\{\lim _{t \rightarrow \infty} \sup \left|y(t)-\theta^{*}\right|\right\}=O\left(a^{2}+1 / \omega^{2}\right) .
\end{aligned}
$$

A demonstração do Teorema 1 segue os passos detalhados nas seções a seguir.

\subsection{Sistema Médio reduzido ODE-PDE}

De (5) e definindo-se

$$
u(x, t)=U(t+x-D),
$$

no qual $t$ é o tempo, $D$ é o atraso e $x$ o estado que permite a seguinte representação como um sistema PDE-ODE:

$$
\begin{gathered}
\dot{\tilde{\theta}}(t-D)=u(0, t), \\
\partial_{t} u(x, t)=\partial_{x} u(x, t), \quad x \in[0, D], \\
u(D, t)=U(t) .
\end{gathered}
$$

Consequentemente, (30) representa a solução do subsistema PDE (33), com (31) sendo uma ODE.

Então, reescreve-se a integral do preditor (26) com o estado da PDE $u(x, t)$ :

$$
U(t)=\frac{c}{s+c}\left\{-k z(t)-k \int_{0}^{D} u(\tau, t) d \tau\right\}
$$

seguindo o mesmo procedimento e utilizando (34), chegase a versão média do sistema ODE-PDE

$$
\begin{gathered}
\dot{\tilde{\theta}}_{a v}(t-D)=u_{a v}(0, t), \\
\partial_{t} u_{a v}(x, t)=\partial_{x} u_{a v}(x, t), \quad x \in[0, D], \\
u_{a v}(D, t)=\frac{c}{s+c}\left\{-k z_{a v}(t)-k \int_{0}^{D} u_{a v}(\tau, t) d \tau\right\} .
\end{gathered}
$$

3.2 Estabilidade Exponencial no Sentido da Norma de Estado Completo

Considera-se a seguinte transformação backstepping de dimensão infinita do estado atrasado 


$$
w(x, t)=u_{a v}(x, t)+\left(k \tilde{\vartheta}_{a v}(t)+k \int_{0}^{x} u_{a v}(\tau, t) d \tau\right),
$$

onde $\vartheta_{a v}(t):=z_{a v}(t)=\tilde{\theta}_{a v}(t-D)$, de acordo com $(20)$. A transformação (38) mapeia o sistema linearizado (35)-(37) em

$$
\begin{gathered}
\dot{\tilde{\vartheta}}_{a v}(t)=-k\left(\tilde{\vartheta}_{a v}(t)+w(0, t)\right), \\
\partial_{t} w(x, t)=\partial_{x} w(x, t), \quad x \in[0, D], \\
\partial_{t} u_{a v}(D, t)=-c w(D, t) .
\end{gathered}
$$

Empregando (35), deriva-se parcialmente o estado transformado $w(x, t)$ em (38) em relação ao tempo $t$ e considerase $x=D$ tal que

$$
\partial_{t} w(D, t)=\partial_{t} u_{a v}(D, t)-k u_{a v}(D, t) .
$$

Além disso, considere a transformação inversa (38)

$$
\begin{array}{r}
u_{a v}(x, t)=w(x, t)+e^{-k x} \tilde{\vartheta}_{a v}- \\
k \int_{0}^{x} e^{-k(x-\tau)} w(\tau, t) d \tau .
\end{array}
$$

Após substituir (41) e (43) em (42), chega-se à

$$
\begin{array}{r}
\partial_{t} w(D, t)=-c w(D, t)+k w(D, t)+ \\
k e^{-k D} \tilde{\vartheta}_{a v}(t)-k^{2} \int_{0}^{D} e^{-k(D-\tau)} w(\tau, t) d \tau .
\end{array}
$$

Dado o seguinte funcional de Lyapunov

$$
\begin{gathered}
V(t)=\frac{\left(\tilde{\vartheta}_{a v}(t)\right)^{2}}{2}-\frac{b}{2} \int_{0}^{D}(1+x) w^{2}(x, t) d x+ \\
\frac{1}{2} w^{2}(D, t),
\end{gathered}
$$

para $b=1 / k$ e $c>0$ suficientemente grande, mostra-se que

$$
\dot{V}(t) \leq \mu V(t)
$$

para algum $\mu>0$. Portanto, O sistema em malha fechada é exponencialmente estável no sentido de norma do estado completo:

$$
\sqrt{\left|\tilde{\vartheta}_{a v}(t)\right|^{2}+w^{2}(D, t)+\int_{0}^{D} w^{2}(x, t) d x}
$$

i.e., na variável transformada $\left(\tilde{\vartheta}_{a v}(t), w(x, t)\right)$.

\subsection{Estabilidade exponencial do Sistema Médio}

Para obter a estabilidade exponencial no sentido de norma

$$
\sqrt{\left|\tilde{\vartheta}_{a v}(t-D)\right|^{2}+\left(U_{a v}(t)\right)^{2}+\int_{t-D}^{t}\left(U_{a v}(\tau)\right)^{2} d \tau},
$$

precisa-se demonstrar que

$$
\alpha_{1} \Psi(t) \leq V(t) \leq \alpha_{2} \Psi(t),
$$

para $\alpha_{1}$ e $\alpha_{2}$ sendo numeros positivos apropriados e

$$
\Psi(t)\left|\tilde{\theta}_{a v}(t-D)\right|^{2}+\left(U_{a v}(t)\right)^{2}+\int_{t-D}^{t}\left(U_{a v}(\tau)\right)^{2} d \tau .
$$

Isso é realizado utilizando uma abordagem similar àquela em (Krstic, 2009, Theorem 1). Assim, obtém-se:

$$
\Psi(t) \leq \frac{\alpha_{2}}{\alpha_{1}} e^{-\mu t} \Psi(0)
$$

que completa a prova da estabilidade exponencial para o sistema médio nas variáveis originais $\left(\tilde{\theta}_{a v}(t-D), u_{a v}(x, t)\right)$.

\subsection{Invocando o Teorema da Média}

Utilizando (5), (26) e (17), obtem-se:

$$
\begin{gathered}
\frac{d}{d t}\left[\begin{array}{c}
\tilde{\theta}(t-D) \\
U(t) \\
\tilde{\Gamma}(t)
\end{array}\right]=\left[\begin{array}{c}
0 \\
-c U(t) \\
0
\end{array}\right]+ \\
-k U(t-D) \\
{\left[\begin{array}{c}
t \\
-c k z(t)-c k \int_{t-D}^{t} U(\tau) d(\tau) \\
\omega_{r}\left(\tilde{\Gamma}(t)+H^{-1}\right)\left(1-\hat{H}(t)\left(\tilde{\Gamma}(t)+H^{-1}\right)\right)
\end{array}\right] .}
\end{gathered}
$$

Define-se o vetor de estado

$$
\mathbf{u}^{\epsilon}(t)=\left[\begin{array}{c}
\tilde{\theta}(t-D) \\
U(t) \\
\tilde{\Gamma}(t)
\end{array}\right]
$$

que geralmente permite expressar (51) na forma de uma equação diferencial funcional estocástica tridimensional

$$
\frac{d}{d t} \mathbf{u}^{\epsilon}(t)=G\left(\mathbf{u}_{t}^{\epsilon}\right)+\epsilon F\left(t, \mathbf{u}_{t}^{\epsilon}, \eta(t), \epsilon\right) .
$$

onde $\epsilon:=1 / \omega$. Portanto, uma vez que $\eta(t)$ é um processo de Markov homogeamente ergódico com medida variante $\mu(d \eta)$ e a propriedade de ergodicidade exponencial, $\mathbf{u}_{t}^{\epsilon}(\delta)=\mathbf{u}^{\epsilon}(t+\delta)$ para $-D \leq \delta \leq 0$ e $G: \mathbf{C}_{3}([-D, 0]) \rightarrow$ $R^{3}$, bem como a função Lipschitz $F: R_{+} \times \mathbf{C}_{3}([-D, 0]) \times$ $Y \times[0,1] \rightarrow R^{3} \operatorname{com} F(t, 0, \eta, \epsilon)=0$ são mapeamentos 
contínuos, pode-se aplicar o teorema da média em (Katafygiotis and Tsarkov, 1999) com seu resultado exponencial de p-estabilidade para o sistema aleatório inicial usando $\epsilon>0$.

\subsection{Convergência Assintótica}

Define-se o tempo de parada (Liu and Krstic, 2010):

$$
\tau_{\epsilon}^{\Delta(\epsilon)}:=\inf \left\{\forall t \geq 0:\left|\mathbf{u}^{\epsilon}(t)\right|>M\left|\mathbf{u}^{\epsilon}(0)\right| e^{-\lambda t}+O(\epsilon)\right\}
$$

como a primeira vez em que a norma do vetor de erro deixa de satisfazer a propriedade de decaimento exponencial. A norma do vetor erro $\left|\mathbf{u}^{\epsilon}(t)\right|$ converge para um valor menor do que o valor residual $\Delta(\epsilon)=O(\epsilon)$. Trata-se de uma convergência exponencial e rápida, que pode se dar de duas formas: almost surely (a.s.) e in probability:

$$
\begin{aligned}
& \lim _{\epsilon \rightarrow 0} \inf \left\{\forall t \geq 0:\left|\mathbf{u}^{\epsilon}(t)\right|>M\left|\mathbf{u}^{\epsilon}(0)\right| e^{-\lambda t}+\Delta\right\}=\infty, \text { a.s. } \\
& \lim _{\epsilon \rightarrow 0} \mathbf{P}\left\{\left|\mathbf{u}^{\epsilon}(t)\right| \leq M\left|\mathbf{u}^{\epsilon}(0)\right| e^{-\lambda t}+\Delta, \forall t \in[0, T(\epsilon)]\right\}=1,
\end{aligned}
$$

com $\lim _{\epsilon \rightarrow 0} \mathrm{~T}(\epsilon)=\infty$. De (55) fica claro que $\tau_{\epsilon}^{\Delta(\epsilon)}$ se aproxima de infinito à medida que $\epsilon$ tende a zero. De forma similar, em (56) a função determinística $T(\epsilon)$ tende a infinito à medida que $\epsilon$ vai a zero. Segue de (55) e (56) que a convergência exponencial é satisfeita dentro de um intervalo de tempo arbitrariamente longo. Qualquer componente do vetor erro converge para um valor menor que $\Delta(\epsilon)=O(\epsilon)$, particularmente o componente $\tilde{\theta}(t)$. Então, pode-se dizer que $\lim _{\epsilon \rightarrow 0} \mathbf{P}\left\{\lim \sup _{t \rightarrow \infty}|\tilde{\theta}(t)|\right\}=$ $O(\epsilon)$. A partir das equações (4) e (8), chega-se a

$$
\theta(t)-\theta^{*}=\tilde{\theta}(t)+a \operatorname{sen}(\eta(t)) .
$$

Uma vez que o primeiro termo no lado direito de (57) é da ordem de $O(\epsilon)$ e o segundo termo é da ordem de $O(a)$, chega-se à equação (28). Finalmente, a partir de (3) e (28), obtém-se (29).

\section{SIMULAÇÕES NUMÉRICAS}

Com o objetivo de avaliar a compensação do atraso no ESC estocástico, considera-se o seguinte mapeamento estático

$$
Q(\theta)=5-\frac{H}{2}(\theta-2)^{2},
$$

aplicando-se uma saída atrasada de $D=100 \mathrm{~s}$. De acordo com (58), o ponto de extremo é $\left(\theta^{*}, y^{*}\right)$ e a Hessiana do mapeamento é $H=-2$. As simulações numéricas do preditor (26) são apresentadas com $C=20, z$ sendo dado por (14) e $G$ em (6), enquanto $\Gamma$ é definido em (15).

Para a relização dos testes, foram utilizados os seguintes parâmetros: $a=0.3, k=0.37, \omega_{r}=0.0052, \Gamma(0)=-\frac{1}{2} \mathrm{e}$ $\theta(0)=0$.
Nas Figuras 2 e 4, encontram-se os resultados gráficos inicialmente na ausência de atrasos, passando pela demonstração da instabilidade quando há a inserção dos atrasos e finalmente, quando utiliza-se o preditor para superar esse problema.

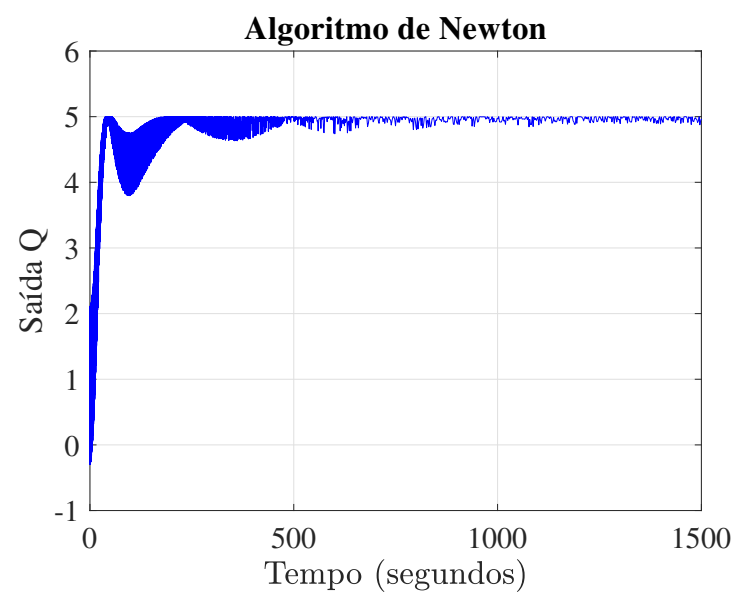

Figura 2. Algoritmo do tipo Newton estocástico na ausência de atrasos.

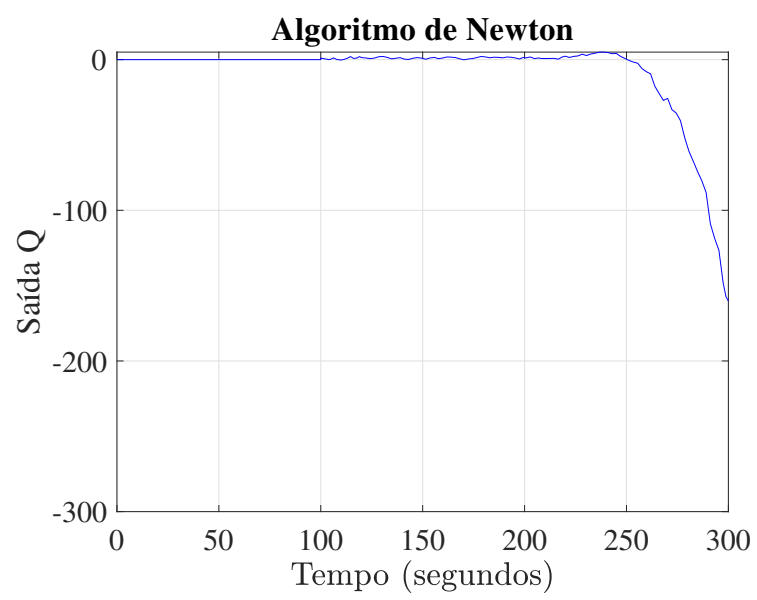

Figura 3. Algoritmo de Newton estocástico na presença de atrasos sem compensação por preditor.

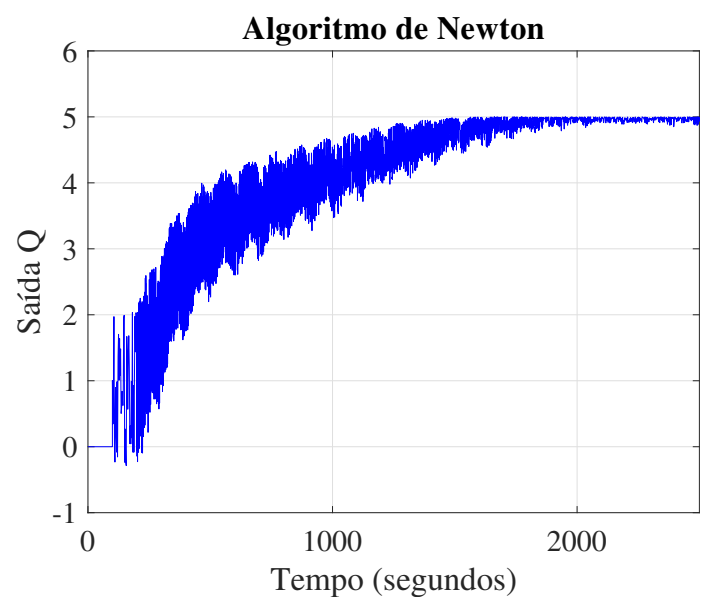

Figura 4. Algoritmo do tipo Newton estocástico na presença de atrasos $D=100 \mathrm{~s}$ e uso do preditor. 
Pela Figura 4, observa-se que a saída do algoritmo de Newton converge para a vizinhança do valor esperado, o sistema em malha fechada permanece estável apesar da existência do atraso que é compensado pelo preditor.

\section{CONCLUSÃO}

Uma nova estratégia de realimentação via preditor baseada em perturbações estocásticas e estimativa da inversa da Hessiana é apresentada para lidar com os atrasos na entrada e/ou saída da malha de controle extremal estocástico, utilizando-se o método de Newton. Essa abordagem preserva a estabilidade exponencial e a convergência da saída do sistema a uma pequena vizinhança do ponto de extremo, mesmo na presença de atrasos. Uma rigorosa demonstração de estabilidade via transformação backstepping e o teorema da média em dimensões infinitas é apresentada. Vale comentar que a taxa de convergência é independente da Hessiana do mapeamento a ser otimizado. Dessa maneira, a compensação dos atrasos pode ser alcançada com uma taxa de convergência atribuída pelo desenvolvedor/usuário, melhorando-se o desempenho do controlador extremal.

\section{REFERÊNCIAS}

Adetola, V. and Guay, M. (2007). Guaranteed parameter convergence for extremum-seeking control of nonlinear systems. Automatica, 43, 105-110.

Ghaffari, A., Krstic, M., and Nesic, D. (2012). Multivariable newton-based extremum seeking. Automatica, 48, 1759-1767.

Hale, J.K. and Lunel, S.M.V. (1990). Averaging in infinite dimensions. Journal of Integral Equations and Applications, 2, 463-494.

Katafygiotis, L. and Tsarkov, Y. (1999). Averaging and stability of quasilinear functional differential equations with Markov parameters. J. Appl. Math. Stochastic Anal., 12, 1-15.

Khalil, H.K. (2002). Nonlinear Systems. Englewood Cliffs: Prentcie-Hall.

Krstic, M. (2008). Lyapunov tools for predictor feedback for delays systems: Inverse optimality and robustness to delays mismatch. Automatica, 44, 2930-2935.

Krstic, M. (2009). Delay Compensation for Nonlinear, Adaptative, and PDE Systems. Birkhauser.

Krstic, M. (2014). Extremum Seeking Control, in T.Samad and J. Baillieul, Encyclopedia of Systems and Control. Springer.

Lehman, B. (2002). The influence of delays when averaging slow and fast oscilations systems: Overview. IMA Journal of Mathematical Control and Information, 19, 201-215.

Liu, S.J. and Krstic, M. (2010). Stochastic averaging in continuous time and its applications in to extremum seeking. IEEE Trans. Autom. Control, 55, 2235-2250.

Liu, S.J. and Krstic, M. (2012). Stochastic Averaging and Stochastic Extremum Seeking. springer.

Mills, G. and Krstic, M. (2018). Maximizing map sensitivity and higher derivatives via extremum seeking. IEEE Transactions on Automatic Control, 63, 3237-3247.

Oliveira, T.R., Hsu, L., and Peixoto, A.J. (2011). Outputfeedback global tracking for unknown control direction plants with applications to extremum-seeking control. Automatica, 47, 2029-2038.

Oliveira, T.R. and Krstic, M. (2015). Newton-based extremum seeking under actuator and sensor delays. IFAC Workshop on Time Delay Systems, 48, 304-309.

Oliveira, T.R., Krstic, M., and Tsubakino, D. (2017). Extremum seeking for static maps with delays. IEEE Transactions on Automatic Control, 62, 1911-1926. 